\title{
The linear constraints in Poincaré and Korn type inequalities *
}

\author{
Giovanni Alessandrini ${ }^{\dagger},{\text { Antonino Morassi }{ }^{\ddagger} \text { and Edi Rosset }}^{\S}$
}

\begin{abstract}
We investigate the character of the linear constraints which are needed for Poincaré and Korn type inequalities to hold. We especially analyze constraints which depend on restriction on subsets of positive measure and on the trace on a portion of the boundary.
\end{abstract}

\section{Introduction}

Let us consider a bounded domain $\Omega \subset \mathbb{R}^{n}$ with Lipschitz boundary. The nature of Poincaré inequality is that a function $u$ (say in $W^{1,2}(\Omega)$ ) is uniquely determined by its gradient $\nabla u \in L^{2}(\Omega)$ up to an additive constant.

Such a constant is usually chosen to be the average of $u$ in $\Omega$, but also the average of its trace on $\partial \Omega$ would do for this purpose. Similarly if one takes a weighted average on suitable subsets $E$ of $\bar{\Omega}$. Meyers $[\underline{6}$ has examined this subject in great depth characterizing the sets $E \subset \bar{\Omega}$ for which this is possible in terms of their capacity. His analysis in fact extends to Poincaré inequalities in $W^{m, p}(\Omega)$, for any $m=1,2, \ldots$ and $1<p<\infty$. See also Meyers and Ziemer [7] for the extreme case of $B V(\Omega)$ and Ziemer [11, Chapter 4] for a more recent account on such results.

In the case of Korn inequality, a vector valued function $u \in W^{1,2}\left(\Omega ; \mathbb{R}^{n}\right)$ is uniquely determined by the strain tensor $\frac{1}{2}\left(\nabla u+(\nabla u)^{T}\right)$ up to an infinitesimal rigid displacement $r(x)=b+M x$, that is an affine transformation for which the matrix $M=\nabla r$ is skew symmetric. In this case, provided the origin of the coordinates is placed in the center of mass of $\Omega, x_{\Omega}=\frac{1}{|\Omega|} \int_{\Omega} x$, an obvious choice is given by $b=\frac{1}{|\Omega|} \int_{\Omega} u, M=\frac{1}{|\Omega|} \int_{\Omega} \frac{1}{2}\left(\nabla u-(\nabla u)^{T}\right)$.

In other words, the common feature of Poincaré and Korn inequalities is that they are inequalities in which a norm is dominated by a seminorm for functions which satisfy a suitable finite set of linear constraints.

In this paper we examine the character of such constraints and in particular we investigate whether such constraints can be chosen in such a way that they can depend only on the values of the function $u$ restricted to a subset of $\Omega$

\footnotetext{
*Work supported in part by MIUR, PRIN n. 2004011204.

$\dagger$ Dipartimento di Matematica e Informatica, Università degli Studi di Trieste, Italy, alessang@univ.trieste.it

¥Dipartimento di Georisorse e Territorio, Università degli Studi di Udine, Italy, antonino.morassi@uniud.it

$\S$ Dipartimento di Matematica e Informatica, Università degli Studi di Trieste, Italy, rossedi@univ.trieste.it
} 
or of its boundary $\partial \Omega$. We are especially interested in obtaining constructive evaluations of the constants in a number of specific cases. In fact, constraints of such type are useful in many instances and in particular, in recent years, they have shown up in connection with inverse boundary value problems, for instance in estimates of unique continuation for the system of elasticity, 2], 8]. For such purposes, it is mandatory to have a concrete and constructive evaluation of the constants involved. In this note we develop this aspect, improving some known estimates, and obtaining some which were not available in the literature.

We deal with three types of inequalities, Poincaré inequality in $W^{1,2}(\Omega)$, Poincaré inequality in $W^{2,2}(\Omega)$ and Korn inequality in $W^{1,2}(\Omega)$. The results are all based on an elementary functional analytic argument (Lemma 2.1) which is due to N. Meyers [6] Proposition 1]. And indeed the consequences for the standard Poincaré inequality, which we summarize in Section 3 are also found in 6.

This is not completely the case for the Poincaré inequality in $W^{2,2}(\Omega)$, Section 4, and Korn inequality, Section 5.

In Section 4 we treat Poincaré inequality in $W^{2,2}(\Omega)$, deriving, along the lines of Meyers' result, the concrete evaluations of the constants in various instances, see Examples 4.2 4.3. We observe however that, when one deals with an open portion $\Gamma$ of the boundary $\partial \Omega$, the suitable linear constraints that occur in Meyers' approach, require the knowledge of the boundary trace of $u$ and of its first derivatives. We thus examine the possibility to determine linear constraints, suitable for the validity of a Poincaré type inequality, which only depend on the trace of $u$ on $\Gamma$. See Example 4.4 and in particular Theorem 4.7 for the Poincaré inequality in $W^{2,2}(\Omega)$. It is peculiar in this case that one has to distinguish the cases when $\Gamma$ is a subset of an $(n-1)$-dimensional hyperplane or not.

In Section 5 we treat Korn inequality and obtain some estimates that we believe to be new. See Examples 5.3 and 5.4 In particular, inequality (5.3) generalizes a classical inequality proven by Kondrat'ev and Oleinik ( 5 , Theorem $1]$ ) when $E$ is a ball and $\Omega$ is starlike with respect to $E$. We also examine the case of constraints which depend on the trace of $u$ on an open portion $\Gamma$ of $\partial \Omega$. In this case, we find that the constraints can be chosen to depend on the trace of $u$ on $\Gamma$ for any open subset $\Gamma$ of $\partial \Omega$, see Theorem [5.7 and Corollary [5.9] which in fact substantially generalize a classical inequality proven by Kondratev and Oleinik ([5] Theorem 2]) for functions $u$ vanishing on a basis of a cylinder $\Omega$.

\section{A basic Lemma}

Lemma 2.1. Let $X, Y$ be Banach spaces, and let $L: X \rightarrow Y$ a bounded linear operator. Let $X_{0} \subset X$ be its null-space and let $P: X \rightarrow X_{0}$ be a bounded linear operator such that $P_{\mid X_{0}}=I d$. Assume that there exists $K>0$ such that

$$
\|x-P x\|_{X} \leq K\|L x\|_{Y}, \quad \text { for every } x \in X .
$$

For every bounded linear operator $T: X \rightarrow X_{0} \subset X$ such that $T_{\mid X_{0}}=I d$ we have

$$
\|x-T x\|_{X} \leq(1+\|T\|) K\|L x\|_{Y}, \quad \text { for every } x \in X .
$$

Here $\|T\|$ denotes the operator norm on $\mathcal{L}(X, X)$. 
Proof. The argument is due to N. Meyers [6] Proposition 1], we reproduce it here, because of its brevity. We have

$$
x-T x=x-P x-T x+P x=x-P x-T(x-P x) .
$$

Hence

$$
\|x-T x\|_{X} \leq(1+\|T\|)\|x-P x\|_{X} \leq(1+\|T\|) K\|L x\|_{Y} .
$$

Remark 2.2. Note that the condition $T_{\mid X_{0}}=I d$ is in fact necessary if an inequality of the following form holds true

$$
\|x-T x\|_{X} \leq \text { Const. }\|L x\|_{Y} .
$$

Indeed, if $x \in X_{0}$, then $L x=0$ and hence $T x=x$. Note also that the evaluation of the constant in (2.2) is not optimal, as is evident if one chooses $T=P$. When $T$ is close to $P$ the following evaluation may be more convenient. Since $(T-P) P x=0$, we have

$$
x-T x=x-P x-(T-P) x=x-P x-(T-P)(x-P x),
$$

hence

$$
\|x-T x\|_{X} \leq(1+\|T-P\|) K\|L x\|_{Y}, \quad \text { for every } x \in X \text {. }
$$

Therefore (2.2) can be improved as follows

$$
\|x-T x\|_{X} \leq(1+\min \{\|T\|,\|T-P\|\}) K\|L x\|_{Y}, \quad \text { for every } x \in X .
$$

\section{The Poincaré inequality}

We shall assume throughout that $\Omega$ is a bounded domain (open and connected) in $\mathbb{R}^{n}$ with Lipschitz boundary $\partial \Omega$.

Definition 3.1. Given a measurable set $E \subset \mathbb{R}^{n}$ with positive measure and given $u \in L^{1}(E)$ we denote its average

$$
u_{E}=\frac{1}{\mu_{n}(E)} \int_{E} u(x) d \mu_{n}(x) .
$$

In the sequel we shall use this notation also for vector and matrix valued functions, in particular we shall denote

$$
x_{E}=\frac{1}{\mu_{n}(E)} \int_{E} x d \mu_{n}(x),
$$

the center of mass of $E$.

We shall use analogous notation for averages on $(n-1)$-dimensional Lipschitz surfaces $\Gamma \subset \mathbb{R}^{n}$

$$
u_{\Gamma}=\frac{1}{\mu_{n-1}(\Gamma)} \int_{\Gamma} u(x) d \mu_{n-1}(x),
$$




$$
x_{\Gamma}=\frac{1}{\mu_{n-1}(\Gamma)} \int_{\Gamma} x d \mu_{n-1}(x) .
$$

Here $\mu_{n-1}$ denotes the $(n-1)$-dimensional Lebesgue measure on $\Gamma$. When no ambiguity occurs we shall also denote $|E|=\mu_{n}(E),|\Gamma|=\mu_{n-1}(\Gamma)$.

We recall the well-known Poincaré inequality.

Theorem 3.2. There exists $Q>0$ such that

$$
\left\|u-u_{\Omega}\right\|_{L^{2}(\Omega)} \leq Q\|\nabla u\|_{L^{2}(\Omega)}, \quad \text { for every } u \in W^{1,2}(\Omega) .
$$

Proof. See, for instance, 10, Theorem 3.6.5]. For a quantitative evaluation of the constant $Q$ in terms of the Lipschitz character of $\Omega$ we refer to [2 Proposition $3.2]$

We also recall the following generalized version, which is a special case of a theorem due to Meyers [6, Theorem 1].

Theorem 3.3. For every $\varphi \in\left(W^{1,2}(\Omega)\right)^{*}$ such that $\varphi(1)=1$ we have

$$
\begin{array}{r}
\|u-\varphi(u)\|_{W^{1,2}(\Omega)} \leq\left(1+\|\varphi\|_{\left(W^{1,2}(\Omega)\right)^{*}}|\Omega|^{\frac{1}{2}}\right) \sqrt{1+Q^{2}}\|\nabla u\|_{L^{2}(\Omega)}, \\
\text { for every } u \in W^{1,2}(\Omega) .
\end{array}
$$

Remark 3.4. Here we have chosen as the $W^{1,2}(\Omega)$-norm the expression

$$
\|u\|_{W^{1,2}(\Omega)}=\left(\int_{\Omega} u^{2}+|\nabla u|^{2}\right)^{\frac{1}{2}} .
$$

Correspondingly, $\|\cdot\|_{\left(W^{1,2}(\Omega)\right)^{*}}$ denotes the dual norm.

Proof of Theorem 3.3. This is in fact a straightforward consequence of Theorem 3.2 and of Lemma 2.1 .

Example 3.5. Let $E$ be any measurable subset of $\Omega$ such that $|E|>0$. By choosing $\varphi(u)=u_{E}$, and computing

$$
|\varphi(u)| \leq \frac{1}{\sqrt{|E|}}\|u\|_{L^{2}(\Omega)} \leq \frac{1}{\sqrt{|E|}}\|u\|_{W^{1,2}(\Omega)}, \quad \text { for every } u \in W^{1,2}(\Omega),
$$

we obtain

$\left\|u-u_{E}\right\|_{W^{1,2}(\Omega)} \leq\left(1+\left(\frac{|\Omega|}{|E|}\right)^{\frac{1}{2}}\right) \sqrt{1+Q^{2}}\|\nabla u\|_{L^{2}(\Omega)}, \quad$ for every $u \in W^{1,2}(\Omega)$.

Example 3.6. Let $\Gamma$ be an open portion of $\partial \Omega$. Let us denote by $\gamma(u)$ the trace on $\Gamma$ of any $u \in W^{1,2}(\Omega)$, and let us choose

$$
\varphi(u)=(\gamma(u))_{\Gamma}
$$

We evaluate

$$
|\varphi(u)| \leq \frac{1}{\sqrt{|\Gamma|}}\|\gamma(u)\|_{L^{2}(\Gamma)} \leq \frac{C_{\Gamma}}{\sqrt{|\Gamma|}}\|u\|_{W^{1,2}(\Omega)}, \quad \text { for every } u \in W^{1,2}(\Omega),
$$


where $C_{\Gamma}$ is the constant in the inequality for the trace imbedding $\gamma: W^{1,2}(\Omega) \rightarrow L^{2}(\Gamma)$ and we obtain

$$
\begin{aligned}
\left\|u-(\gamma(u))_{\Gamma}\right\|_{W^{1,2}(\Omega)} \leq\left(1+C_{\Gamma}\left(\frac{|\Omega|}{|\Gamma|}\right)^{\frac{1}{2}}\right) \sqrt{1+Q^{2}}\|\nabla u\|_{L^{2}(\Omega)}, \\
\quad \text { for every } u \in W^{1,2}(\Omega) .
\end{aligned}
$$

\section{A higher order Poincaré inequality}

We treat an analogue of Theorem 3.3 suitable for functions in $W^{2,2}(\Omega)$.

We denote by $\mathcal{A}$ the space of affine functions on $\Omega$

$$
\mathcal{A}=\left\{u(x)=a+b \cdot x \mid a \in \mathbb{R}, b \in \mathbb{R}^{n}\right\} .
$$

The following Theorem is again a special case of Theorem 1 in $[6]$. We report it here with a slightly different expression of the constant on the right hand side.

Theorem 4.1. For any bounded operator $T: W^{2,2}(\Omega) \rightarrow \mathcal{A}$ such that $T u=u$ for every $u \in \mathcal{A}$, we have

$\|u-T u\|_{W^{2,2}(\Omega)} \leq(1+\|T\|)\left(1+Q^{2}+Q^{4}\right)^{\frac{1}{2}}\left\|\nabla^{2} u\right\|_{L^{2}(\Omega)}, \quad$ for every $u \in W^{2,2}(\Omega)$.

Here $Q$ is the constant for the Poincaré inequality (3.5).

Proof. The proof is immediate, by a repeated application of Poincaré inequality (3.5) and using Lemma 2.1

Example 4.2. In analogy to Example 3.5 given $E \subset \Omega$ measurable, with $|E|>0$, we pose

$$
T u=u_{E}+(\nabla u)_{E} \cdot\left(x-x_{E}\right) .
$$

Note that $T$ is uniquely determined by the restriction of $u$ to $E$. In fact if $u_{1}$, $u_{2} \in W^{1,2}(\Omega)$ are such that $\left.u_{1}\right|_{E}=\left.u_{2}\right|_{E}$ then $\nabla\left(u_{1}-u_{2}\right)=0$ almost everywhere in $E$ (see [4] Lemma 7.7]).

Clearly $T$ is the identity on $\mathcal{A}$ and we compute

$$
\|T u\|_{W^{2,2}(\Omega)}^{2} \leq 2 \frac{|\Omega|}{|E|}\left(1+(\operatorname{diam} \Omega)^{2}\right)\|u\|_{W^{1,2}(\Omega)}^{2}
$$

and consequently we obtain

$$
\begin{aligned}
\left\|u-\left(u_{E}+(\nabla u)_{E} \cdot\left(x-x_{E}\right)\right)\right\|_{W^{2,2}(\Omega)} \leq \\
\leq\left(1+\left(2 \frac{|\Omega|}{|E|}\left(1+(\operatorname{diam} \Omega)^{2}\right)\right)^{\frac{1}{2}}\right)\left(1+Q^{2}+Q^{4}\right)^{\frac{1}{2}}\left\|\nabla^{2} u\right\|_{L^{2}(\Omega)}, \\
\quad \text { for every } u \in W^{2,2}(\Omega) .
\end{aligned}
$$

Example 4.3. In certain instances, it is useful to have an estimate of the type (4.3) when $\Omega$ and $E$ are concentric balls, say $\Omega=B_{1}(0), E=B_{\rho}(0), 0<\rho<1$. In this case we evaluate

$$
\|T\| \leq \sqrt{\frac{n+3}{n+2}}\left(\frac{1}{\rho}\right)^{\frac{n}{2}}
$$


Consequently

$$
\begin{gathered}
\|u-T u\|_{W^{2,2}\left(B_{1}(0)\right)} \leq\left(1+\sqrt{\frac{n+3}{n+2}}\left(\frac{1}{\rho}\right)^{\frac{n}{2}}\right)\left(1+Q^{2}+Q^{4}\right)^{\frac{1}{2}}\left\|\nabla^{2} u\right\|_{L^{2}\left(B_{1}(0)\right)}, \\
\text { for every } u \in W^{2,2}\left(B_{1}(0)\right)
\end{gathered}
$$

This specific estimate turns out to be useful in an inverse problem for elastic plates, 9.

Example 4.4. We note that in general it is not possible to mimic Example 3.6 for a Poincaré type inequality in $W^{2,2}(\Omega)$ when some functionals of the trace of $u$ alone on an open portion $\Gamma$ of the boundary are known.

For example, if one considers

$$
\Omega=\left\{x \in \mathbb{R}^{n} \mid 0<x_{i}<1, i=1, \ldots, n\right\}
$$

and

$$
\Gamma=\left\{x \in \mathbb{R}^{n} \mid x_{n}=0,0<x_{i}<1, i=1, \ldots, n-1\right\},
$$

then the function $u=x_{n}$ has zero trace on $\Gamma$ and $\left\|\nabla^{2} u\right\|_{L^{2}(\Omega)}=0$. Hence its $W^{2,2}(\Omega)$-norm is not dominated by $\left\|\nabla^{2} u\right\|_{L^{2}(\Omega)}$ and any functional of its trace on $\Gamma$.

In fact some additional assumptions, or data, are necessary.

If we admit that $\Gamma$ may be flat, additional pieces of information are needed, see [6]. For instance, if also the trace of $\nabla u$ on $\Gamma$ is available, then one can consider

$$
T u=(\gamma(u))_{\Gamma}+(\gamma(\nabla u))_{\Gamma} \cdot\left(x-x_{\Gamma}\right)
$$

and obtain

$$
\begin{array}{r}
\left\|u-(\gamma(u))_{\Gamma}-(\gamma(\nabla u))_{\Gamma} \cdot\left(x-x_{\Gamma}\right)\right\|_{W^{2,2}(\Omega)} \leq(1+\|T\|)\left(1+Q^{2}+Q^{4}\right)^{\frac{1}{2}}\left\|\nabla^{2} u\right\|_{L^{2}(\Omega)} \\
\text { for every } u \in W^{2,2}(\Omega)
\end{array}
$$

And we compute

$$
\|T\| \leq C_{\Gamma}\left(2 \frac{|\Omega|}{|\Gamma|}\left(1+(\operatorname{diam} \Omega)^{2}\right)\right)^{\frac{1}{2}}
$$

where $C_{\Gamma}$ is the constant in the inequality for the trace imbedding $\gamma: W^{1,2}(\Omega) \rightarrow L^{2}(\Gamma)$.

Let us assume, instead, that $\Gamma \subset \partial \Omega$ is not flat, that is, it is not a portion of an hyperplane. In this case we obtain Theorem 4.7 below. In order to state it, we first need some preparation.

We choose a reference system whose origin lies on the center of mass of $\Gamma$, that is, we assume

$$
x_{\Gamma}=0 .
$$

Lemma 4.5. Assume that $\Gamma$ is not a portion of an hyperplane. The restriction to $\mathcal{A}$ of the trace imbedding $\gamma: W^{1,2}(\Omega) \rightarrow L^{2}(\Gamma)$ is one to one.

Proof. First of all we observe that, in view of (4.7), $\Gamma$ is not a portion of an hyperplane if and only if there exists $n$ linearly independent vectors $v_{1}, \ldots, v_{n} \in$ 
$\Gamma$. Thus, if $l(x)=a+b \cdot x \in \mathcal{A}$ and $\gamma(l)=0$, then $a=(\gamma(l))_{\Gamma}=0$ and $b \in \mathbb{R}^{n}$ must satisfy

$$
b \cdot v_{i}=\gamma(l)\left(v_{i}\right)-a=0, \text { for every } i=1, \ldots, n .
$$

Consequently $b=0$ and hence, $l=0$.

Remark 4.6. Observe that, being $\Omega$ bounded, any sufficiently large portion $\Gamma$ of the boundary shall not be flat.

Let us denote by $e: \gamma(\mathcal{A}) \rightarrow \mathcal{A}$ the inverse of $\left.\gamma\right|_{\mathcal{A}}$, and by $\pi: L^{2}(\Gamma) \rightarrow \gamma(\mathcal{A})$ the orthogonal projection onto $\gamma(\mathcal{A})$ with respect to the $L^{2}(\Gamma)$ inner product. Note that an explicit expression of $\pi$ is easily obtained. In fact, for every $v \in L^{2}(\Gamma), \pi(v)$ is determined as $\pi(v)=\gamma(l)$, where $l \in \mathcal{A}$ is the minimizer of the finite dimensional least squares problem

$$
\min \left\{\int_{\Gamma}(v-l)^{2} \mid l \in \mathcal{A}\right\} .
$$

We also introduce $\tau: L^{2}(\Gamma) \rightarrow \mathcal{A}$ as the composition $\tau=e \circ \pi$.

Theorem 4.7. Assume that $\Gamma$ is not a portion of an hyperplane. We have

$$
\begin{array}{r}
\|u-\tau(\gamma(u))\|_{W^{2,2}(\Omega)} \leq\left(1+C_{\Gamma}\|e\|\right)\left(1+Q^{2}+Q^{4}\right)^{\frac{1}{2}}\left\|\nabla^{2} u\right\|_{L^{2}(\Omega)} \\
\quad \text { for every } u \in W^{2,2}(\Omega) .
\end{array}
$$

Here $\|e\|$ denotes the $\mathcal{L}\left(L^{2}(\Gamma), W^{2,2}(\Omega)\right)$-norm of $e, C_{\Gamma}$ is the constant in the inequality for the trace imbedding $\gamma: W^{1,2}(\Omega) \rightarrow L^{2}(\Gamma)$ and $Q$ is the constant for the Poincaré inequality (3.5).

Remark 4.8. Observe that the calculation of the norm $\|e\|$ reduces to solving a finite dimensional eigenvalue problem, in fact

$$
\|e\|^{2}=\max \left\{\left.\frac{\int_{\Omega}(a+b \cdot x)^{2}+|b|^{2}}{\int_{\Gamma}(a+b \cdot x)^{2}}\left|a \in \mathbb{R}, b \in \mathbb{R}^{n}, a^{2}+\right| b\right|^{2}>0\right\} .
$$

As a consequence of Theorem 4.7 we obtain the following Corollary.

Corollary 4.9. If $\Gamma$ is not a portion of an hyperplane, then we have

$$
\|u\|_{W^{2,2}(\Omega)} \leq C_{1}\left\|\nabla^{2} u\right\|_{L^{2}(\Omega)}+C_{2}\|\gamma(u)\|_{L^{2}(\Gamma)}, \quad \text { for every } u \in W^{2,2}(\Omega),
$$

where $C_{1}, C_{2}>0$ are constants depending on $\Omega$ and $\Gamma$ only.

Proof of Theorem 4.7. It suffices to apply Theorem 4.1 with $T=\tau \circ \gamma$ and to observe that, since $\|\pi\|_{\mathcal{L}\left(L^{2}(\Gamma), L^{2}(\Gamma)\right)}=1$, then we have $\|T\| \leq C_{\Gamma}\|\tau\| \leq C_{\Gamma}\|e\|$.

\section{$5 \quad$ Korn type inequalities}

We recall the Korn inequality (of second kind). Given $u \in W^{1,2}\left(\Omega ; \mathbb{R}^{n}\right)$ we denote

$$
\widehat{\nabla} u=\frac{1}{2}\left(\nabla u+(\nabla u)^{T}\right) .
$$


Theorem 5.1. There exists $K>0$ such that

$$
\left\|\nabla u-\frac{1}{2}\left(\nabla u-(\nabla u)^{T}\right)_{\Omega}\right\|_{L^{2}(\Omega)} \leq K\|\widehat{\nabla} u\|_{L^{2}(\Omega)}, \quad \text { for every } u \in W^{1,2}\left(\Omega ; \mathbb{R}^{n}\right) \text {. }
$$

Proof. See for instance [3].

We denote by Skew ${ }^{n}=\left\{M \in \mathbb{M}^{n \times n} \mid M^{T}=-M\right\}$ the class of skewsymmetric $n \times n$ matrices and by $\mathcal{R}=\left\{b+M x \mid b \in \mathbb{R}^{n}, M \in\right.$ Skew $\left.^{n}\right\}$ the linear space of infinitesimal rigid displacements.

Theorem 5.2. For any bounded linear operator $T: W^{1,2}\left(\Omega ; \mathbb{R}^{n}\right) \rightarrow \mathcal{R}$ such that $T u=u$ for every $u \in \mathcal{R}$, we have

$\|u-T u\|_{W^{1,2}(\Omega)} \leq(1+\|T\|) K\left(1+Q^{2}\right)^{\frac{1}{2}}\|\widehat{\nabla} u\|_{L^{2}(\Omega)}, \quad$ for every $u \in W^{1,2}\left(\Omega ; \mathbb{R}^{n}\right)$.

Here $K$ is the constant appearing in (5.1) and $Q$ is the constant in the Poincaré inequality (3.5).

Proof. Set $P: W^{1,2}\left(\Omega ; \mathbb{R}^{n}\right) \rightarrow \mathcal{R}$ as

$$
P u=u_{\Omega}+\frac{1}{2}\left(\nabla u-(\nabla u)^{T}\right)_{\Omega}\left(x-x_{\Omega}\right) .
$$

By (3.5) and (5.1) we obtain

$$
\|u-P u\|_{W^{1,2}(\Omega)} \leq K\left(1+Q^{2}\right)^{\frac{1}{2}}\|\widehat{\nabla} u\|_{L^{2}(\Omega)} .
$$

The thesis follows from Lemma 2.1 by choosing

$$
\begin{gathered}
X=W^{1,2}\left(\Omega ; \mathbb{R}^{n}\right), \\
Y=L^{2}\left(\Omega ; \mathbb{M}^{n \times n}\right), \\
L u=\widehat{\nabla} u, \\
X_{0}=\mathcal{R} .
\end{gathered}
$$

Example 5.3. Given $E \subset \Omega$ measurable with $|E|>0$ and setting $T u=u_{E}+$ $\frac{1}{2}\left(\nabla u-(\nabla u)^{T}\right)_{E}\left(x-x_{E}\right)$, we compute

$$
\|T u\|_{W^{1,2}(\Omega)}^{2} \leq 2 \frac{|\Omega|}{|E|}\left(1+(\operatorname{diam} \Omega)^{2}\right)\|u\|_{W^{1,2}(\Omega)}^{2} .
$$

Consequently, we obtain

$$
\begin{aligned}
\left\|u-u_{E}-\frac{1}{2}\left(\nabla u-(\nabla u)^{T}\right)_{E}\left(x-x_{E}\right)\right\|_{W^{1,2}(\Omega)} & \leq \\
\leq\left(1+\left(2 \frac{|\Omega|}{|E|}\left(1+(\operatorname{diam} \Omega)^{2}\right)\right)^{\frac{1}{2}}\right) & K\left(1+Q^{2}\right)^{\frac{1}{2}}\|\widehat{\nabla} u\|_{L^{2}(\Omega)}, \\
& \text { for every } u \in W^{1,2}\left(\Omega ; \mathbb{R}^{n}\right) .
\end{aligned}
$$


Example 5.4. When $\Omega$ and $E$ are concentric balls, say $\Omega=B_{1}(0), E=B_{\rho}(0)$, $0<\rho<1$. In this case we evaluate

$$
\|T\| \leq \sqrt{\frac{n+3}{n+2}}\left(\frac{1}{\rho}\right)^{\frac{n}{2}} .
$$

Consequently

$$
\begin{array}{r}
\|u-T u\|_{W^{1,2}\left(B_{1}(0)\right) \leq}\left(1+\sqrt{\frac{n+3}{n+2}}\left(\frac{1}{\rho}\right)^{\frac{n}{2}}\right) K\left(1+Q^{2}\right)^{\frac{1}{2}}\|\widehat{\nabla} u\|_{L^{2}\left(B_{1}(0)\right)}, \\
\text { for every } u \in W^{1,2}\left(B_{1}(0) ; \mathbb{R}^{n}\right) .
\end{array}
$$

This bound improves the one in [1, Lemma 3.5] in two respects. It applies to any dimension and the exponent of $\frac{1}{\rho}$ is diminished.

Example 5.5. We examine a version of Korn inequality when constraints on $u$ are taken on its trace on an open portion $\Gamma$ of $\partial \Omega$. The following Lemma will be useful.

Lemma 5.6. The restriction to $\mathcal{R}$ of the trace imbedding $\gamma: W^{1,2}\left(\Omega ; \mathbb{R}^{n}\right) \rightarrow L^{2}\left(\Gamma ; \mathbb{R}^{n}\right)$ is one to one.

Proof. With no loss of generality we assume $x_{\Gamma}=0$. Being $\Gamma$ an $(n-1)-$ dimensional hypersurface, there exist $v_{1}, \ldots, v_{n-1} \in \Gamma$ which are linearly independent. We show that, given $r \in \mathcal{R}$ such that $\gamma(r)=0$ we have $r=0$. In fact we have $r(0)=\gamma(r)_{\Gamma}=0$ and also $r\left(v_{i}\right)=\gamma(r)\left(v_{i}\right)=0$ for every $i=1, \ldots, n-1$. It is well known that such constraints imply $r=0$. We provide a proof for the sake of completeness. Let us write $r(x)=b+M x$ with $b \in \mathbb{R}^{n}$ and $M \in$ Skew $^{n}$. We have $b=r(0)=0$, hence it remains to prove that the conditions $r\left(v_{i}\right)=0, i=1, \ldots, n-1$ imply $M=0$. Up to a rotation in the reference system, we can assume that the $n$-th component of the vectors $v_{i}$, $i=1, \ldots, n-1$, is zero. Thus we have

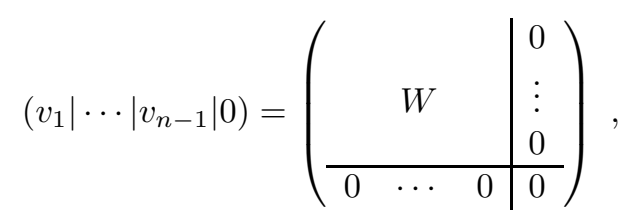

where $W$ is a nonsingular $(n-1) \times(n-1)$ matrix. We have

$$
M v_{i}=r\left(v_{i}\right)=0 \text {, for every } i=1, \ldots, n-1 \text {. }
$$

Consequently

$$
M\left(\begin{array}{ccc|c} 
& & & 0 \\
& W & & \vdots \\
& & & 0 \\
\hline 0 & \cdots & 0 & 0
\end{array}\right)=0
$$

and also

$$
M\left(\begin{array}{ccc|c} 
& & 0 \\
& I & & \vdots \\
& & & 0 \\
\hline 0 & \cdots & 0 & 0
\end{array}\right)=M\left(\begin{array}{ccc|c} 
& & & 0 \\
& W & & \vdots \\
& & & 0 \\
\hline 0 & \cdots & 0 & 0
\end{array}\right)\left(\begin{array}{lll|l} 
& & 0 \\
& W^{-1} & \vdots \\
& & & 0 \\
\hline 0 & \cdots & 0 & 0
\end{array}\right)=0,
$$


where $I$ is the $(n-1) \times(n-1)$ identity matrix. It follows that the entries below the diagonal of $M$ are all zero and, consequently, $M=0$.

Let us denote by $E: \gamma(\mathcal{R}) \rightarrow \mathcal{R}$ the inverse of $\left.\gamma\right|_{\mathcal{R}}$, and by $\Pi: L^{2}\left(\Gamma ; \mathbb{R}^{n}\right) \rightarrow \gamma(\mathcal{R})$ the orthogonal projection onto $\gamma(\mathcal{R})$ with respect to the $L^{2}\left(\Gamma ; \mathbb{R}^{n}\right)$ inner product. We also introduce $\rho: L^{2}\left(\Gamma ; \mathbb{R}^{n}\right) \rightarrow \mathcal{R}$ as the composition $\rho=E \circ \Pi$.

Theorem 5.7. Given an open portion $\Gamma$ of $\partial \Omega$, we have

$$
\begin{array}{r}
\|u-\rho(\gamma(u))\|_{W^{1,2}(\Omega)} \leq\left(1+C_{\Gamma}\|E\|\right) K\left(1+Q^{2}\right)^{\frac{1}{2}}\|\widehat{\nabla} u\|_{L^{2}(\Omega)}, \\
\text { for every } u \in W^{1,2}\left(\Omega ; \mathbb{R}^{n}\right) .
\end{array}
$$

Here $\|E\|$ denotes the $\mathcal{L}\left(L^{2}\left(\Gamma ; \mathbb{R}^{n}\right), W^{1,2}\left(\Omega ; \mathbb{R}^{n}\right)\right)$-norm of $E, C_{\Gamma}$ is the constant in the inequality for the trace imbedding $\gamma: W^{1,2}(\Omega) \rightarrow L^{2}(\Gamma)$ and $Q$ is the constant for the Poincaré inequality (3.5).

Remark 5.8. Arguing as in Remark 4.8] also the calculation of the norm $\|E\|$ reduces to solving a finite dimensional eigenvalue problem.

The proof is straightforward, as well as the one of the following Corollary.

Corollary 5.9. There exist $C_{1}, C_{2}>0$, depending only on $\Omega$ and $\Gamma$, such that

$$
\|u\|_{W^{1,2}(\Omega)} \leq C_{1}\|\widehat{\nabla} u\|_{L^{2}(\Omega)}+C_{2}\|\gamma(u)\|_{L^{2}(\Gamma)}, \quad \text { for every } u \in W^{1,2}\left(\Omega ; \mathbb{R}^{n}\right)
$$

Acknowledgement. The authors wish to thank an anonymous referee for providing fundamental bibliographical information which was missing in a previous version of this paper.

\section{References}

[1] G. Alessandrini, A. Morassi and E. Rosset. Detecting an inclusion in an elastic body by boundary measurements. SIAM J. Math. Anal. 33 (6) (2002) $1247-1268$.

[2] G. Alessandrini, A. Morassi and E. Rosset. Detecting cavities by electrostatic boundary measurements. Inverse Problems 18 (2002) 1333-1353.

[3] K.O. Friedrichs. On the boundary value problems of the theory of elasticity and Korn's inequality. Annals of Math. 48 (1947) 441-471.

[4] D. Gilbarg and N.S. Trudinger. Elliptic partial differential equations of second order. Springer, New York, 1983.

[5] V.A. Kondrat'ev and O.A. Oleinik. On the dependence of the constant in Korn's inequality on parameters characterizing the geometry of the region. Russian Math. Surveys 44 (1989) 187-195.

[6] N.G. Meyers. Integral inequalities of Poincaré and Wirtinger type. Arch. Rational Mech. Anal. 68 (2) (1978) 113-120. 
[7] N.G. Meyers and W.P. Ziemer. Integral inequalities of Poincaré and Wirtinger type for BV functions. Amer. J. Math. 99 (6) (1977) 1345-1360.

[8] A. Morassi and E. Rosset. Uniqueness and stability in determining a rigid inclusion in an elastic body. Submitted for publication.

[9] A. Morassi, E. Rosset and S. Vessella. Size estimates for inclusions in an elastic plate by boundary measurements. Submitted for publication.

[10] C.B. Morrey. Multiple Integrals in the Calculus of Variations. SpringerVerlag, New York, 1966.

[11] W.P. Ziemer. Weakly Differentiable Functions. Springer-Verlag, New York, 1989. 